\title{
ESTUDO DE CASO ACERCA DA DISSEMINAÇÃO DA TECNOLOGIA BIM POR MEIO DE ENSINO REMOTO
}

José Matheus Anacleto Caldeira Alves-jmatheusalves3@gmail.com

Universidade Federal de Alagoas, Centro de Tecnologia

Maceió - Alagoas

Fernanda Ferreira Albuquerque Tenorio-fernanda.tenorio@ctec.ufal.br

Universidade Federal de Alagoas, Centro de Tecnologia

Maceió-Alagoas

Alícia Caroline de Lima Silva-alicia.lima@ctec.ufal.br

Universidade Federal de Alagoas, Centro de Tecnologia

Maceió - Alagoas

Karoline Alves de Melo Moraes - kamm@ctec.ufal.br

Universidade Federal de Alagoas, Centro de Tecnologia

Maceió - Alagoas

\section{Resumo}

Executar com qualidade e eficiência um empreendimento de Arquitetura e Engenharia tem se tornado uma tarefa complexa. Para cumprir as exigências de um mercado cada vez mais rigoroso, um novo paradigma se apresenta: a tecnologia BIM. Nesse contexto, o presente estudo tem como objetivo apresentar a experiência do PET Engenharia Civil da Universidade Federal de Alagoas na realização de cursos sobre a ferramenta BIM 3D, em particular através do software REVIT, que, no contexto atual de isolamento social, foi ministrado de modo remoto, potencializando o uso dessa modalidade de aprendizado. A partir dos dados obtidos, observou-se que o conhecimento nas plataformas abordadas ainda não está disseminado e que a atividade conseguiu um forte impacto em abrandar esse cenário.

Palavras-chave: Cursos. Ensino Remoto. BIM.

\section{CASE STUDY ABOUT THE DISSEMINATION OF THE BIM TECHNOLOGY BY REMOTE EDUCATION}

\section{Abstract:}

Executing architectural and engineering constructions with quality and efficiency has become an increasingly complex task. To attend the demands of an increasingly strict market, a new paradigm is presented: BIM technology. In this context, this study aims to present the experience of PET Engenharia Civil from the Federal University of Alagoas in the remote teaching of BIM 3D, in particular through the REVIT software, in the current context of social isolation, which enhances the use of this learning modality. Through the data obtained, it was observed that the knowledge in the addressed platforms is not yet disseminated and that the activity achieves a strong impact in mitigating this scenario.

Keywords: Courses. Remote Teaching. BIM 


\section{INTRODUÇÃO}

O avanço da indústria da Arquitetura, Engenharia e Construção (AEC) no país está diretamente conectado à gestão da qualidade nos seus setores de projeto (HIPPERT e ARAÚJO, 2010). Chegar mais perto a esse objetivo está relacionado ao progresso das Tecnologias da Informação (TI) que trazem maior precisão e agilidade a esse processo.

Executar um empreendimento de Arquitetura e Engenharia envolve diversas necessidades de controle, como "informações sobre especificações de materiais, custos de mão de obra, tempo de execução, compatibilização das instalações de infraestrutura, entre outros" (PEREIRA e RIBEIRO, 2014). Observa-se nitidamente que projetar em Computer Aided Design (CAD) não contempla mais essas necessidades, nem o rigor dos, cada vez mais complexos, empreendimentos atuais, principalmente no tocante ao gerenciamento, representação e interoperabilidade (BASTO e LORDSLEEM JUNIOR, 2016). Para atingir esse novo patamar, cada vez mais empresas do ramo estão utilizando a Modelagem da Informação da Construção, do inglês Building Information Modeling (BIM).

O BIM se apresenta como uma representação digital baseada na modelagem dos objetos e das características físicas e funcionais de uma obra, de forma inteligente e paramétrica, replicando assim as relações que irão existir, após a execução, entre todos os elementos (BAPTISTA, 2015). Nesse contexto, o BIM não se apresenta como um mero avanço tecnológico, mas sim como uma mudança de paradigma na forma de apresentar e representar a informação da construção (GODOY et al., 2013).

Diferente do modelo 3D em softwares $\mathrm{CAD}$, que apenas representa a forma dos objetos, no modelo BIM é possível associar esse modelo a outras informações quantitativas e paramétricas. Essas informações permitem posteriormente a geração de representações bidimensionais e relatórios que permitirão a realização de análises da qualidade e desempenho, além de orçamentos e cronogramas.

Na contramão a essa realidade cada vez mais latente, ainda há bastante desinformação sobre o BIM entre os alunos e professores do ensino superior, sendo um conceito ainda embrionário nas universidades como visto, por exemplo, no estudo de Junior e Pincerato (2017). Incluir este tema nas grades curriculares já é premente uma vez que, como afirmado por Catelani (2016), a tecnologia BIM só tende a crescer e se consolidar na indústria da construção.

Outro tipo de avanço tecnológico diz respeito à necessidade de difusão do BIM. A maior acessibilidade às Tecnologias Digitais de Informação e Comunicação (TDIC) e o atual contexto de hiperconectividade trouxeram uma revolução na forma de armazenar e disseminar o conhecimento (KEMCZINSKI et. al, 2000). Assim sendo, as formas de ensinoaprendizagem remotas já se apresentam para o suporte e auxílio às instituições e organizações no processo de formação dos profissionais.

Entretanto, embora essa realidade aporte uma série de benefícios, como a flexibilização do ambiente de aprendizagem para os envolvidos, esta também requere destes uma contrapartida. Os estudantes devem estar dispostos a incorporar em seu cotidiano um processo organizacional e de gestão de suas atividades, que permita que desenvolvam uma aprendizagem autônoma. Esse fato se apresenta como um empecilho para a efetividade desta modalidade.

No entanto, o contexto atual, com o isolamento social promovido pela pandemia do Covid-19, acelerou a inserção e ampliação do uso de estratégias de ensino remoto no dia-a-dia das organizações, estratégias essas que afinal se encaixam totalmente na atipicidade do momento.

Sendo assim, o presente trabalho tem o objetivo de apresentar a experiência do PET Engenharia Civil da UFAL na disseminação do conhecimento sobre o BIM, em particular no 
software REVIT, através de minicursos na modalidade à distância. Esses minicursos fizeram parte de uma atividade gratuita e aberta a toda a comunidade, elaborada pelo grupo no contexto da pandemia, com o propósito de suprir a ausência de atividades acadêmicas neste período. Com os dados obtidos, é possível entender tanto a realidade atual do conhecimento dessas tecnologias no público atingido, como também o impacto da atividade em mitigar o incipiente acesso a estas.

\section{METODOLOGIA}

$\mathrm{Na}$ atividade em questão foram ofertados três cursos relacionados à tecnologia BIM, todos utilizando o software Revit: projetos arquitetônicos, hidrossanitários e elétricos. A criação da ementa de cada curso foi feita por seus respectivos ministrantes, que elaboraram as aulas em forma de vídeos que, posteriormente, foram disponibilizados no YouTube com configuração de visibilidade "não-listado", permitindo que apenas aqueles que se inscreveram nas capacitações pudessem acessar o conteúdo dos vídeos. A escolha pela disponibilização dos cursos de maneira gravada, em vez de ao vivo, se deu para facilitar a aprendizagem dos inscritos, visto que, nesse caso, há a possibilidade de pausas e replays de acordo com as necessidades de cada participante.

Todos os cursos foram estruturados de modo que os projetos fossem desenvolvidos de maneira lógica e executados pelos participantes à medida que os vídeos fossem assistidos, para que estes fossem submetidos à avaliação dos ministrantes, como ferramenta de comprovação da participação efetiva na capacitação. Sendo assim, foi necessário definir um prazo de duração, período no qual os vídeos estiveram disponíveis para acesso, de maneira que os inscritos tivessem tempo suficiente para acompanhar satisfatoriamente os cursos e pudessem adequá-los ao dia-a-dia, o que possibilitou que mais pessoas fossem beneficiadas. Por isso, as capacitações referentes aos projetos arquitetônico e hidrossanitários ficaram disponíveis para os alunos por um período de uma semana, enquanto que o curso referente à parte elétrica foi disponibilizado por quatorze dias.

Ademais, foi observada a necessidade da criação de um canal direto entre ministrantes e inscritos, para que todas as dúvidas pudessem ser sanadas. Por isso, ao início de cada curso, foram criados grupos no aplicativo WhatsApp, para que os participantes pudessem elucidar suas dúvidas de forma imediata.

Os conteúdos abordados nos cursos foram divididos em duas partes, sendo uma teórica e outra prática. Em se tratando da parte teórica, os ministrantes realizaram uma introdução breve à tecnologia BIM e ao software utilizado e trataram a teoria pertinente a cada projeto em si, tornando as capacitações acessíveis para estudantes que ainda não cursaram matérias referentes aos assuntos e àqueles que ainda não haviam tido contato com o Revit. Já na parte prática foi desenvolvido todo o projeto proposto.

Desse modo, os cursos permitiram aos concluintes o desenvolvimento de conhecimentos técnicos, relacionados à representação de elementos arquitetônicos, dimensionamento de tubulações e de condutores elétricos, orientações e recomendações de projeto, além das habilidades relativas à utilização eficiente do software, para seus devidos fins. 
Para os participantes que finalizaram os cursos foi disponibilizado um formulário de avaliação da atividade, cujas perguntas podem ser vistas na Tabela 1.

Tabela 1 - Perguntas apresentadas para a avaliação de cada curso.

\begin{tabular}{|l|l|l|}
\hline $\begin{array}{l}\text { Seção 1: Nível de } \\
\text { Aprendizado }\end{array}$ & Seção 2: Conteúdos do Curso & $\begin{array}{l}\text { Seção 3: Escolha do } \\
\text { Curso }\end{array}$ \\
\cline { 1 - 2 } $\begin{array}{l}\text { Conhecimento no início do } \\
\text { curso }\end{array}$ & Os objetivos foram alcançados? & $\begin{array}{l}\text { Por que você escolheu } \\
\text { esse curso? }\end{array}$ \\
\cline { 1 - 2 } $\begin{array}{l}\text { Conhecimento no fim do } \\
\text { curso }\end{array}$ & $\begin{array}{l}\text { O conteúdo do curso foi organizado } \\
\text { e bem planejado? }\end{array}$ & \\
\cline { 1 - 2 } $\begin{array}{l}\text { Contribuição do curso para } \\
\text { o dia-a-dia }\end{array}$ & $\begin{array}{l}\text { A carga horária do curso foi } \\
\text { apropriada? }\end{array}$ & \\
\hline
\end{tabular}

Fonte - Os Autores

\section{RESULTADOS E DISCUSSÃO}

Para os resultados acerca do curso de Revit arquitetônico foram avaliadas as respostas dos formulários de feedback das duas turmas realizadas. É importante ressaltar que a certificação não estava interligada à avaliação do curso, desse modo, foram obtidas 76 respostas espontâneas, o que corresponde a, aproximadamente, $67 \%$ do total de participantes que concluíram o curso e enviaram o projeto finalizado para correção.

As respostas para as duas primeiras perguntas da seção "Nível de aprendizado" para o curso de Revit arquitetônico, expostas no Gráfico 1, permitem um estudo comparativo entre o conhecimento dos inscritos no início e final do curso. É perceptível que o nível de evolução do conhecimento foi significativo, visto que, no início do curso, cerca de $54 \%$, dos alunos que responderam o formulário tinham um conhecimento ruim ou moderado sobre o assunto abordado no curso, em comparação com, aproximadamente, $97 \%$ de respostas dos alunos que consideraram seu conhecimento final como "Excelente", "Muito Bom" ou "Satisfatório".

Além disso, ainda no Gráfico 1, podemos perceber o grau de exigência da tecnologia BIM para o dia a dia dos inscritos, visto que aproximadamente $92 \%$ dos alunos responderam que a contribuição do curso, para seu cotidiano, foi "Excelente" ou "Muito Bom".

Gráfico 1 - Respostas da seção "Nível de Aprendizado" para as duas turmas do curso "Revit Arquitetônico".

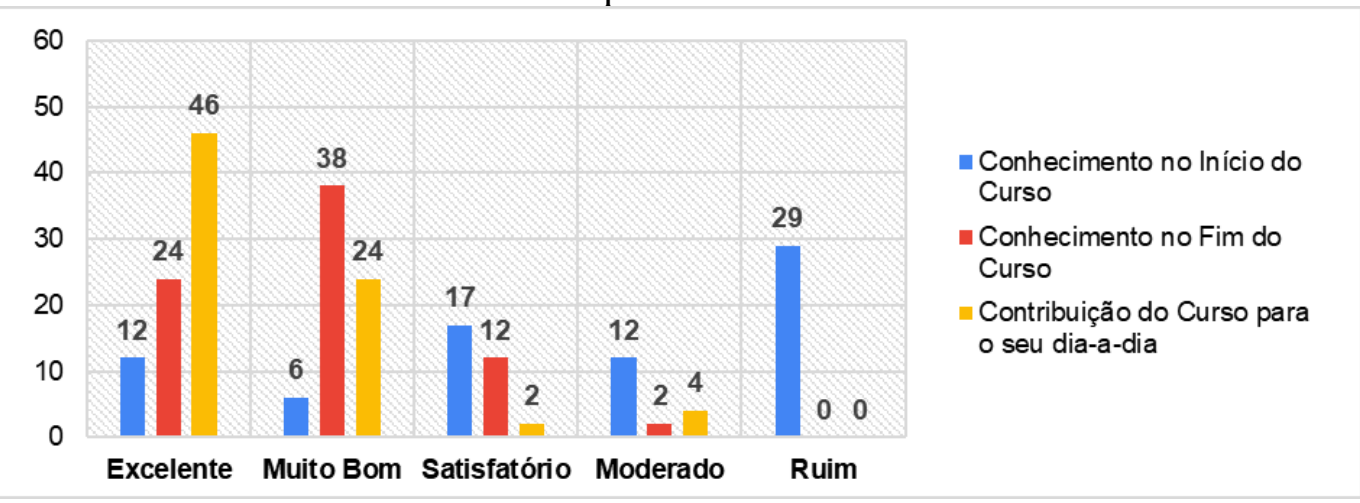

Fonte - Os Autores 
Já as respostas obtidas e expostas no Gráfico 2, referentes ao conteúdo abordado no decorrer do curso de Revit Arquitetônico, evidenciam que todos os inscritos se mostraram satisfeitos com o que foi proposto em relação aos objetivos, à organização e planejamento e à carga horária do curso, visto que apenas uma pessoa se absteve quanto à pergunta sobre a carga horária do curso.

Gráfico 2 - Respostas da seção "Conteúdos do Curso" para as duas turmas do curso "Revit Arquitetônico".

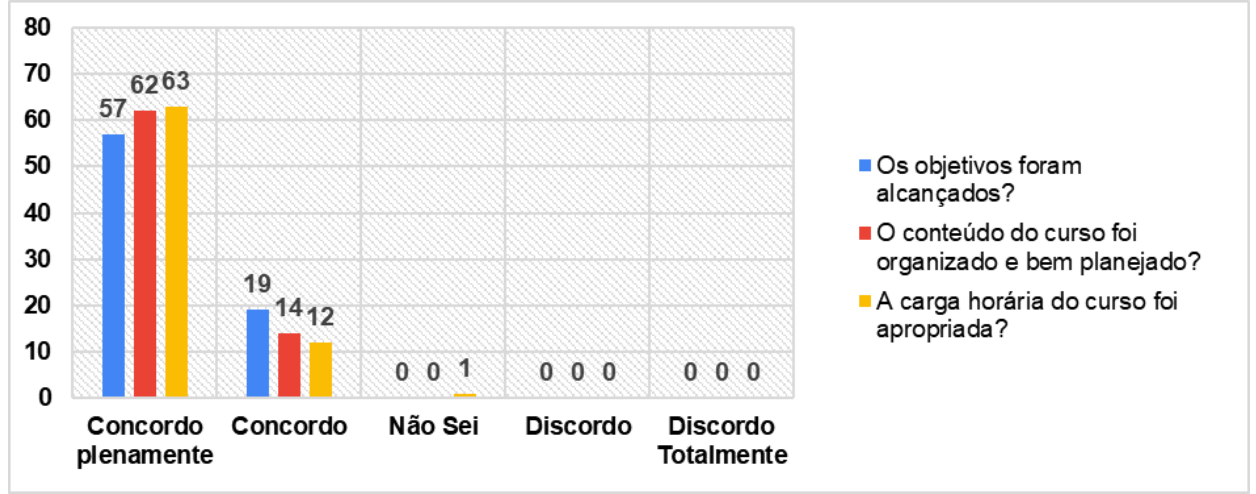

Fonte - Os Autores

O primeiro dos dois cursos que abordaram projetos complementares tratou sobre Projetos Hidrossanitários no Revit. Buscou-se explanar não só a modelagem dessas estruturas no software, mas também o básico sobre dimensionamento e o funcionamento das instalações de água fria e sanitárias.

Essa abordagem aumenta a especificidade desse curso, retratada diretamente na alta porcentagem de pessoas que consideravam seu conhecimento no início do curso como "ruim", $64,3 \%$ das respostas obtidas. Além disso, é possível notar o impacto do curso ministrado em amenizar essa condição, através da sondagem do conhecimento no fim do curso dos participantes, com 67,8\% deles considerando-o "muito bom" ou "excelente". As respostas da seção "Nível de Aprendizado" estão expostas no Gráfico 3.

Nas respostas da seção "Conteúdo do Curso" é possível inferir que os objetivos propostos pela ementa abordada foram atingidos com $75,8 \%$ das respostas apontando plena concordância nas duas primeiras perguntas. A única ressalva vai ao tempo dado para realização do curso (uma semana) que não se mostrou adequado para o tamanho da carga horária proposta, sendo sugerido um tempo maior em uma futura turma. As respostas para essa seção estão apresentadas no Gráfico 4.

Gráfico 3 - Respostas da seção "Nível de Aprendizado" para as duas turmas do curso "Revit: Projetos Hidrossanitários".

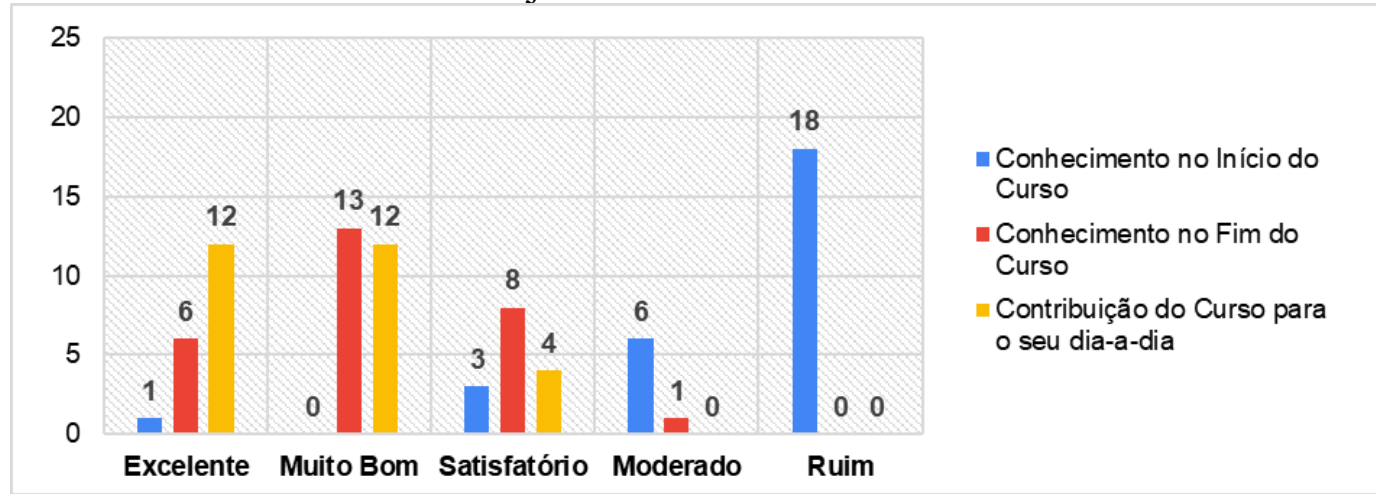

Fonte - Os Autores 
Gráfico 4 - Respostas da seção "Conteúdos do Curso" para as duas turmas do curso "Revit: Projetos Hidrossanitários".

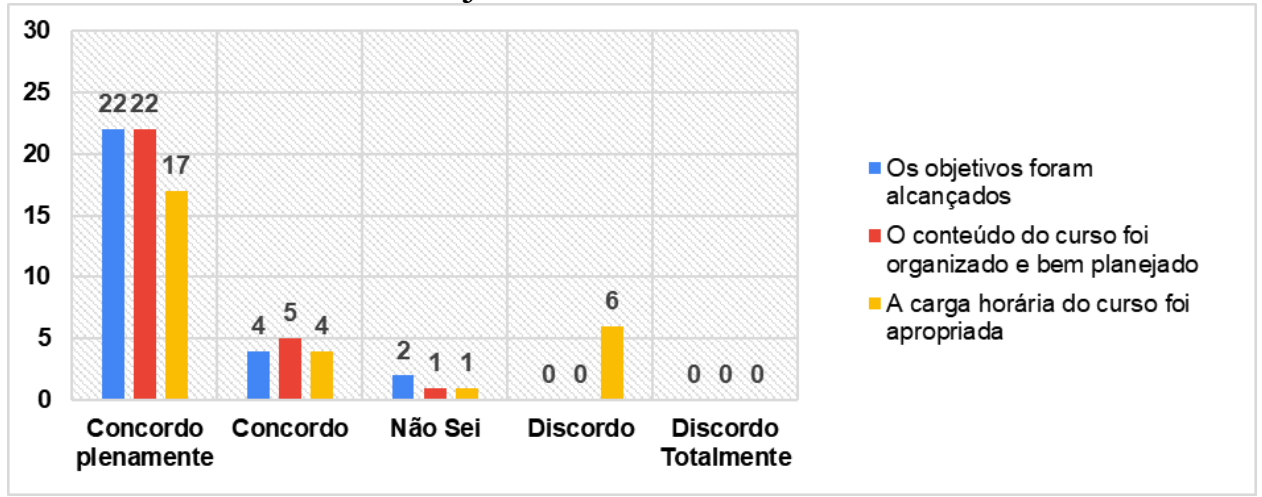

Fonte - Os Autores

No tocante aos projetos complementares, o segundo curso abordado foi o "Revit: Projetos Elétricos". Conectado ao mesmo princípio de abordar também aspectos teóricos dos projetos elétricos, a ementa tratou não só a modelagem desse sistema no programa.

Foi possível observar mais uma vez que grande parte dos participantes considerava o seu conhecimento no início do curso como "ruim", dessa vez 45,9\% das respostas. Esse cenário foi transformado após o curso com 83,8\% dos participantes considerando seu conhecimento no fim do curso como "muito bom" ou "excelente". Os dados da seção "Nível de Aprendizado" para este curso podem ser visualizados no Gráfico 5.

Gráfico 5 - Respostas da seção "Nível de Aprendizado" para as duas turmas do curso "Revit: Projetos Elétricos".

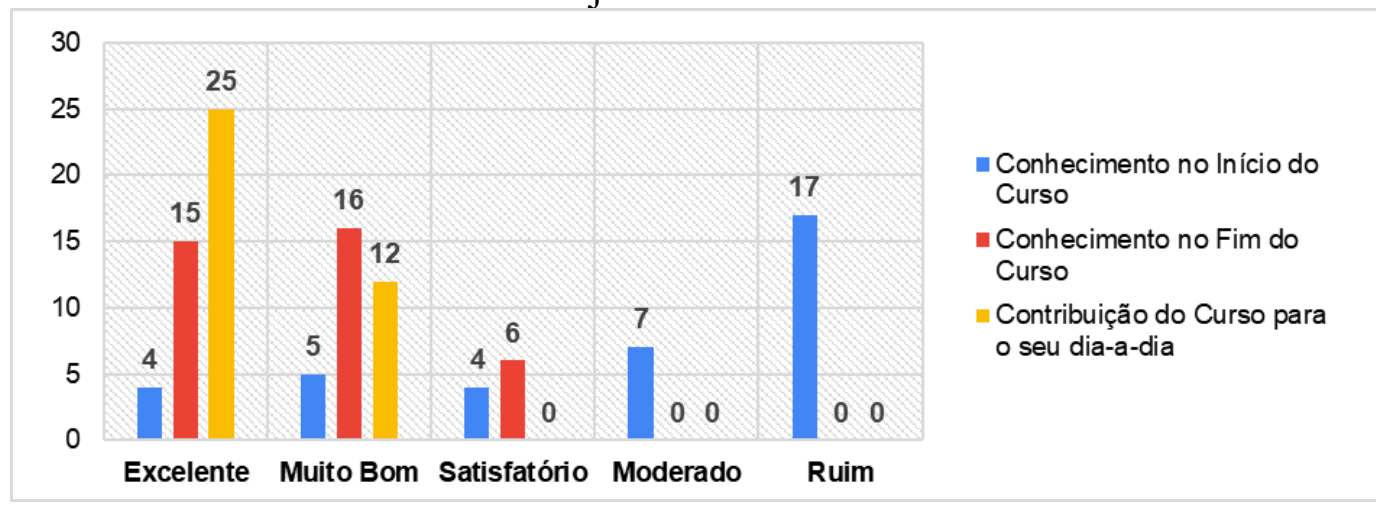

Fonte - Os Autores

Quanto à seção "Conteúdos do Curso", é possível notar que a ementa foi bem aceita pelos participantes, com mais de $80 \%$ das respostas concordando plenamente tanto na pergunta "Os objetivos foram alcançados?", como na "O conteúdo do curso foi organizado e bem planejado?".

De posse das sugestões vindas do curso "Revit: Projetos Hidrossanitários" foi feita uma maior harmonização entre a carga horária e o tempo de realização do curso, que agora foi de duas semanas. Isso resultou diretamente em uma melhor avaliação do público na pergunta "A carga horária do curso foi apropriada?", com nenhuma resposta "Discordo" ou "Discordo Totalmente". Os dados dessa seção estão expostos no Gráfico 6. 
Gráfico 6 - Respostas da seção "Conteúdos do Curso" para as duas turmas do curso "Revit:

Projetos Elétricos".

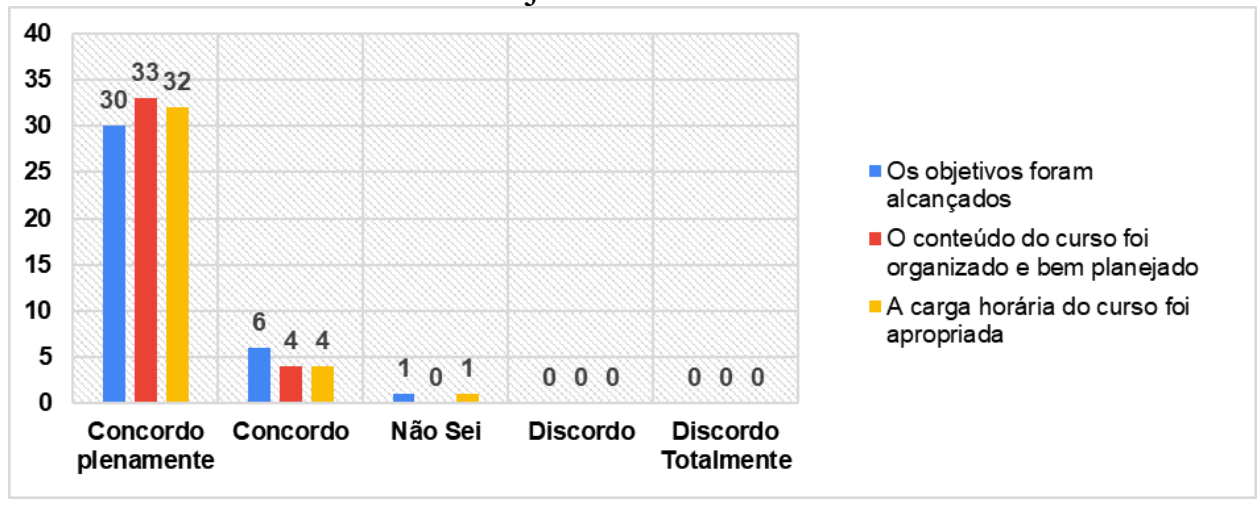

Fonte - Os Autores

O Gráfico 7 apresenta as respostas dos participantes quanto à motivação de participação nos três cursos em questão.

Gráfico 7 - Respostas da pergunta "Por que você escolheu esse curso?" para todos os cursos.

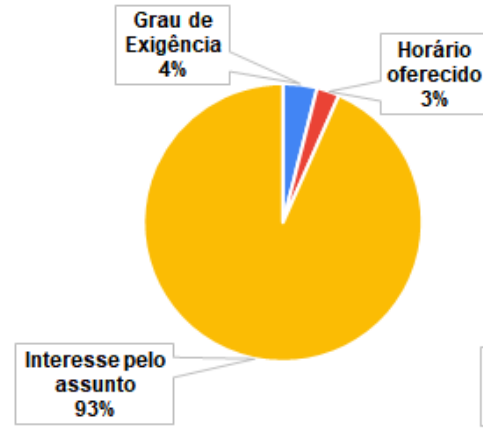

Revit Arquitetônico

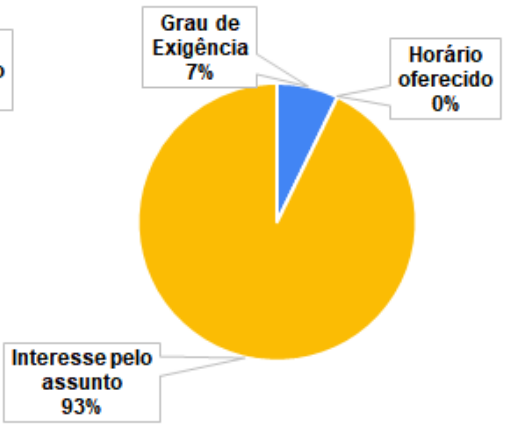

Revit Projetos Hidrossanitários

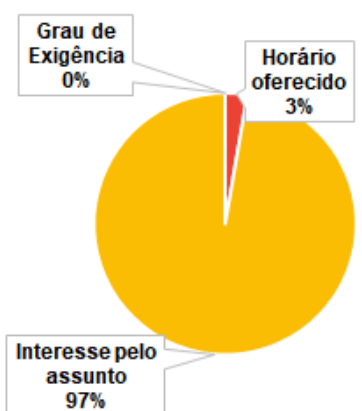

Revit Projetos Elétricos

- Grau de Exigência - Horário oferecido = Interesse pelo assunto

Fonte - Os Autores

Pode-se perceber que, para o "Revit: Arquitetônico", 93\% dos que responderam o formulário participaram do curso por interesse no assunto. $\mathrm{O}$ mesmo ocorre em relação aos cursos dos projetos complementares ofertados, o "Projetos Elétricos" pode ser destacado por essa porcentagem chegar a 97\%. É perceptível, portanto, que o entusiasmo dos estudantes e profissionais da área de engenharia vem crescendo cada vez mais, fazendo com que busquem se especializar na tecnologia BIM e nos softwares que a envolvem.

\section{CONCLUSÕES}

A partir dos resultados apresentados no tocante à comparação entre o conhecimento no início e no final do curso, pode-se afirmar que a grande maioria dos participantes apresentava um nível de conhecimento baixo sobre o software e o BIM. Isso confirma, mais uma vez que, esse aprendizado precisa ser mais explorado nas Instituições de Ensino Superior, mais especificamente para os cursos relacionados à área de Construção Civil, de forma a trabalhar e colocar em prática essas habilidades. 
Ademais, é possível perceber, com base no feedback e respostas obtidas, que as ementas e o formato dos cursos foram eficientes e bem aceitos, de acordo com a seção "Conteúdos do Curso", bem como a análise do conhecimento ao fim da atividade e a contribuição para o dia a dia.

Por fim, é importante ressaltar que há curiosidade e disposição por parte dos estudantes para a participação de cursos que abordem essas temáticas, dado que a grande maioria se inscreveu pelo interesse no assunto. Portanto, sugere-se que atividades similares devem continuar sendo realizadas, dentro e fora das IES, para que o ensino de BIM seja cada vez mais difundido, em suas diversas formas e especificidades.

\section{Agradecimentos}

Os autores agradecem à Secretaria de Educação Superior (Sesu/MEC), pela concessão de bolsa através do Programa de Educação Tutorial - PET.

\section{REFERÊNCIAS}

BAPTISTA, A. Utilização de Ferramentas BIM no Planeamento de Trabalhos de Construção - Estudo de Caso. Dissertação de Mestrado. Faculdade de Engenharia da Universidade do Porto, 2015.

BASTO, P. E. de A.; LORDSLEEM JUNIOR, A. C. O ensino de BIM em curso de graduação em engenharia civil em uma universidade dos EUA: estudo de caso. Ambiente Construído, Porto Alegre, v. 16, n. 4, p. 45-61, out./dez. 2016.

CATELANI, W.S. et al. Implementação do BIM para Construtoras e Incorporadoras. v.1 ao v.6. Brasília: CBIC, 2016.

GODOY, V.; CARDOSO, C.; BORGES, M. BIM: desafios para um conceito em construção no ensino de arquitetura e engenharia. In: CONGRESSO BRASILEIRO DE EDUCAÇÃO EM ENGENHARIA, 41., Gramado, 2013. Anais... Gramado: ABENGE, 2013.

HIPPERT, M. A. S; ARAÚJO, T. T. BIM e a qualidade do projeto: Um estudo de caso em uma pequena empresa de projeto. In: XIII ENTAC (Encontro Nacional de Tecnologia do Ambiente Construído). Anais... Canela: ANTAC, 2010.

JUNIOR, R.; PINCERATO, G. Importância da disseminação do ensino do BIM nas universidades e implementação dentro de construtoras para formação de BIM Managers. Colloquium Exactarum. 9. p. 129-135, 2017

KEMCZINSKI, A. et al. O desempenho e a satisfação discente em um modelo de ensinoaprendizagem semi-presencial. In: XXVIII Congresso Brasileiro de Ensino de Engenharia, 2000, Ouro Preto. Anais... Ouro Preto: ABENGE, 2000.

PEREIRA, P.; RIBEIRO, R. A Inserção do BIM no Curso de Graduação em Engenharia Civil. In: CONGRESSO BRASILEIRO DE EDUCAÇÃO EM ENGENHARIA, 42., Juiz de Fora, 2014. Anais... Juiz de Fora: ABENGE, 2014. 